\title{
Definition, Risikofaktoren und Klassifikationsmodelle von Pseudarthrosen
}

Karl Friedrich Braun, Marc Hanschen, Peter Biberthaler

\section{Einleitung}

Die Knochenbruchheilung stellt einen deutlich längeren und komplexeren Heilungsprozess im Vergleich zu anderen Geweben im Körper dar. Die Besonderheit liegt in der Tatsache begründet, dass der Wiederaufbau des Knochens mit denselben biomechanischen und biochemischen Eigenschaften und ohne minderwertige fibröse Narbe stattfindet und somit eine Restitutio ad integrum möglich ist [1,2]. Dieser Heilungsprozess ist ein Ineinandergreifen von unterschiedlichen zellulären Abläufen, die vereinfacht zusammengefasst beginnend mit dem Frakturhämatom über nicht mineralisierten und mineralisierten Kallus bis hin zu kraftlinienorientiertem Remodeling abläuft. Eine zunehmende Belastungsstabilität ist i.d.R. nach 6 Wochen zu erwarten, wobei je nach frakturiertem Knochen und Lokalisation diese Heilungszeit deutlich variieren kann. ( $\triangleright$ Tab. 1) [3].

Die Knochenheilung muss als dynamischer Prozess gesehen werden. Neben den klassischen Grundregeln der Knochenbruchheilung „je jünger, umso rascher“ und „Von oben nach unten“ gelten die in > Tab. 1 genannten ossären Konsolidierungszeiten daher nur als Richtwerte, um eine eventuelle verzögerte Knochenbruchheilung zu detektieren. Sie dürfen nicht als pauschale Heilungszeit missverstanden werden.

Die Herausforderungen „wenn der Knochen nicht heilt“ sind groß. In ca. 5-10\% aller Frakturen kommt es zu einer verzögerten Knochenbruchheilung mit Ausbildung einer Pseudarthrose, was allein für die BRD eine Fallzahl zwischen 40000 und 80000 Patienten bedeutet [4,5]. Bestehen individuelle Risikofaktoren beim Patienten, kann diese Rate rasch auf bis zu 30\% ansteigen ( $\triangleright$ Abb. 2) [6, 7]. Der Altersgipfel dieser schwerwiegenden Komplikation liegt zwischen dem 30. und 40. Lebensjahr, was neben der medizinischen vor allem die sozioökonomische Relevanz unterstreicht $[5,9,10]$. Die Behandlungsverlängerung durch eine verzögerte Knochenheilung stellt eine wesentliche Beeinträchtigung der Lebensqualität dar und verlängert die Krankheitsdauer entscheidend. Persistierende Belastungsschmerzen (insbesondere bei Biegeund Rotationsstress), Überwärmung und Überempfindlichkeit der Haut auf Frakturhöhe sowie eingeschränkte Funktionstüchtigkeit müssen den behandelnden Arzt an eine fehlerhaft und/oder verzögert ablaufende Knochenbruchheilung denken lassen.

Im Folgenden soll auf die Begriffsverwirrung bei der Definition der verzögerten Knochenbruchheilung im deutschen Sprachgebrauch eingegangen sowie die Risikofaktoren der Pseudarthrosenentstehung herausgearbeitet werden. Ferner werden die gängigen Klassifikationsmodelle der verzögerten Knochenbruchheilung dargestellt, die aktuell jedoch nur bedingt eine therapeutische Konsequenz aufweisen.

\section{Definition}

Es gibt keine weltweite klare und vor allem exakte Definition der Pseudarthrose. Weder der Zeitpunkt, ab wann von einer verzögerten Knochenheilung ausgegangen wird, noch ab wann konkret von einer Pseudarthrose gesprochen wird, ist bisher einheitlich definiert. Insbesondere herrscht eine Begriffsverwirrung zwischen dem angloamerikanischen und dem deutschen Sprachgebrauch. Zeigt sich eine ausbleibende Knochenbruchheilung über einen Zeitraum von 4 bis 6 Monaten, so wird dies in Deutschland als verzögerte Knochenbruchheilung be-

- Tab. 1 Heilungszeiten der unterschiedlichen Knochen [3].

\begin{tabular}{|l|l|}
\hline Knochenbruch & Heilungszeit (Wochen) \\
\hline Klavikula & $3-8$ \\
\hline Skapula & 6 \\
\hline Rippen & 4 \\
\hline Humerus & $4-10$ \\
\hline Radius, Ulna & 6 \\
\hline Handgelenk & $4-12$ \\
\hline Finger & $4-6$ \\
\hline Becken & $4-6$ \\
\hline Femur & 12 \\
\hline Patella & $4-6$ \\
\hline Tibia, Fibula & $10-24$ \\
\hline Sprunggelenk & 6 \\
\hline Fuß & $3-12$ \\
\hline Zehe & 3 \\
\hline
\end{tabular}


- Tab. 2 Interindividuelle Risikofaktoren [5].

\begin{tabular}{|l|l|}
\hline patientenabhängige Risikofaktoren & $\begin{array}{l}\text { patientenunabhängige } \\
\text { Risikofaktoren }\end{array}$ \\
\hline Alter & Frakturlokalisation \\
\hline Geschlecht & Frakturspaltweite $(>2 \mathrm{~mm})$ \\
\hline Komorbiditäten & Frakturtyp \\
\hline Knochenstoffwechselerkrankungen & Weichteilschaden \\
\hline Infektion & Knochendefekte \\
\hline Rauchen & Osteosyntheseverfahren \\
\hline Alkohol & \\
\hline Ernährung & \\
\hline Medikamente & \\
\hline
\end{tabular}

zeichnet. Die ausbleibende knöcherne Konsolidierung nach 6 Monaten wird sodann im deutschen Sprachgebrauch als Pseudarthrose definiert $[5,13]$. Dieser rigiden Betrachtungsweise steht eine etwas dynamischere Definition der FDA (US Food and Drug Administration) gegenüber. Von einer „delayed union“ (verzögerten Knochenbruchheilung) wird bei fehlender radiologischer und klinischer Heilungstendenz nach 3 Monaten gesprochen, während eine „non-union“ erst nach ausbleibender ossärer Konsolidierung nach 9 Monaten vorliegt. Jedoch liegt auch im Angloamerikanischen der Hauptfokus auf dem zeitlichen Verlauf, was die interindividuellen Patientengegebenheiten und Einflussfaktoren nicht ausreichend berücksichtigt ( $\triangleright$ Tab. 1, 2 und 3).

Losgelöst von der zeitlichen Pauschalierung definiert die AO (Arbeitsgemeinschaft für Osteosynthesefragen) das Vorliegen einer Pseudarthrose, wenn der behandelnde Arzt keine Heilungstendenz mehr erkennen kann und die vorliegende Fraktur nicht ohne erneute Intervention zur Ausheilung kommt und einer fokussierten und akkuraten Therapie bedarf (z.B. Reosteosynthese, Anfrischung, Knochentransplantation). Diese Betrachtungsweise entspricht am ehesten der anspruchsvollen Dynamik der Knochenbruchheilung und der Vielzahl an Einflussfaktoren, birgt jedoch entsprechend dem Erfahrungsschatz des behandelnden Arztes Risiken hinsichtlich der zeitnahen und progressiven Diagnosestellung. Somit ergibt sich als Wegweiser für eine Definition aktuell nur ein Kompromiss. Der Zeitraum für eine physiologische Frakturheilung trotz unterschiedlicher Heilungs- und Regenerationspotenziale der betroffenen Knochen sollte 3-4 Monate nicht überschreiten. Nach 4-8 Monaten muss bei fehlender ossärer Konsolidierung von einer verzögerten Frakturheilung ausgegangen werden und nach 9 Monaten von einer Pseudarthrose gesprochen werden. Entscheidend für den behandelnden Arzt ist jedoch die Kombination aus Röntgenverlaufskontrollen der Fraktur und dem klinischen Bild des Patienten mit Blick auf die Heilungstendenz und nicht allein auf die Heilungsdauer.
- Tab. 3 Pseudarthrosenrate verschiedener Röhrenknochen. Quelle: Schmidmaier G, Moghaddam A. Pseudarthrosen langer Röhrenknochen. Z Orthop Unfall 2015; 153 : 659-676.

\begin{tabular}{|l|c|}
\hline Röhrenknochen & Pseudarthroserate \\
\hline Tibia & $45 \%$ \\
\hline Femur & $16 \%$ \\
\hline Humerus & $9 \%$ \\
\hline Ulna & $7 \%$ \\
\hline Radius & $5 \%$ \\
\hline
\end{tabular}

\section{Risikofaktoren einer verzögerten Knochenheilung}

Sowohl patientenabhängige als auch-unabhängige Risikofaktoren sind mit einer Häufung der verzögerten Knochenbruchheilung vergesellschaftet. Hervorzuheben ist hier vor allem das Rauchen mit einer signifikant verlängerten Frakturheilung [7, 8]. Weiterhin birgt die jeweilige Frakturlokalisation ein unterschiedliches Risikoprofil zur Ausbildung einer Pseudarthrose. Die Häufigkeit des Auftretens variiert in Abhängigkeit vom betroffenen Knochen basierend auf u.a. speziellen Besonderheiten wie der ossären Blutversorgung (Os scaphoideum, Talus, medialer Schenkelhals etc.; $>$ Tab. 3).

Im Allgemeinen sind diaphysäre Frakturen häufiger von einer Pseudarthrose betroffen als metaphysäre Frakturen. Gleiches gilt für offene Knochenbrüche bzw. Frakturen mit erheblichem Weichteilschaden und/oder Knochenverlust - hier steigt das Pseudarthrosenrisiko deutlich an. Weiterhin treten Pseudarthrosen an der unteren Extremität häufiger auf als an der oberen Extremität mit der höchsten Rate bei Tibiaschaftfrakturen. Dies liegt u.a. an dem geringen Weichteilmantel und der schlechten Durchblutungssituation sowie den häufig schwerwiegenden und offenen Frakturen [11]. Es wird ersichtlich, dass mögliche Störfaktoren in der kaskadenartig ablaufenden Knochenbruchheilung vielfältig sind und daher einer dezidierten und professionellen Analyse bedürfen. Ihre Behandlung gehört daher in die Hände eines erfahrenen Kollegen (Pseudarthrosensprechstunde) [3, 12].

Als prädiktives Tool zur Risikoeinschätzung des individuellen Pseudarthroserisikos ist das in > Tab. 4 dargestellte, von Moghaddam und Mitarbeitern entworfene ScoringSystem sehr nützlich, um rechtzeitig mögliche Komplikationen vorherzusehen und eine notwendige Therapie nicht zu verzögern [7]. 
- Tab. 4 Score zur Einschätzung des individuellen Risikos der Entstehung einer Pseudarthrose. Quelle: Schmidmaier G, Moghaddam A. Pseudarthrosen langer Röhrenknochen. Z Orthop Unfall 2015; 153: 659-676.

\begin{tabular}{|c|c|c|c|}
\hline Risikofaktor & \multicolumn{3}{|l|}{ Lokalisation/Bewertung } \\
\hline \multicolumn{4}{|l|}{ Röhrenknochen } \\
\hline Humerus & $\begin{array}{l}\text { proximal } \\
4 \text { Punkte }\end{array}$ & $\begin{array}{l}\text { diaphysär } \\
6 \text { Punkte }\end{array}$ & $\begin{array}{l}\text { distal } \\
2 \text { Punkte }\end{array}$ \\
\hline Unterarm & $\begin{array}{l}\text { proximal } \\
4 \text { Punkte }\end{array}$ & $\begin{array}{l}\text { diaphysär } \\
6 \text { Punkte }\end{array}$ & $\begin{array}{l}\text { distal } \\
2 \text { Punkte }\end{array}$ \\
\hline Femur & $\begin{array}{l}\text { proximal } \\
4 \text { Punkte }\end{array}$ & $\begin{array}{l}\text { diaphysär } \\
6 \text { Punkte }\end{array}$ & $\begin{array}{l}\text { distal } \\
8 \text { Punkte }\end{array}$ \\
\hline Tibia & $\begin{array}{l}\text { proximal } \\
6 \text { Punkte }\end{array}$ & $\begin{array}{l}\text { diaphysär } \\
8 \text { Punkte }\end{array}$ & $\begin{array}{l}\text { distal } \\
4 \text { Punkte }\end{array}$ \\
\hline \multicolumn{4}{|l|}{ Weichteile } \\
\hline & $\begin{array}{l}\text { erstgradig offene Weichteil- } \\
\text { verletzung } \\
4 \text { Punkte }\end{array}$ & $\begin{array}{l}\text { zweitgradig offene Weichteil- } \\
\text { verletzung } \\
6 \text { Punkte }\end{array}$ & $\begin{array}{l}\text { drittgradig offene Weichteil- } \\
\text { verletzung } \\
10 \text { Punkte }\end{array}$ \\
\hline & $\begin{array}{l}\text { Fasziotomie } \\
4 \text { Punkte }^{\mathrm{a}}\end{array}$ & $\begin{array}{l}\text { vorausgegangene Fraktur } \\
8 \text { Punkte }^{\text {a }}\end{array}$ & $\begin{array}{l}\text { neurologische Schäden } \\
6 \text { Punkte }\end{array}$ \\
\hline \multicolumn{4}{|l|}{ weitere Risikofaktoren } \\
\hline Rauchen & $\begin{array}{l}\text { Raucher } \\
15 \text { Punkte }\end{array}$ & $\begin{array}{l}\text { früherer Raucher } \\
5 \text { Punkte }\end{array}$ & $\begin{array}{l}\text { Nichtraucher } \\
0 \text { Punkte }\end{array}$ \\
\hline Komorbiditäten/Medikamente & $\begin{array}{l}\text { NSAID } \\
4 \text { Punkte }\end{array}$ & $\begin{array}{l}\text { Bisphosphonate } \\
6 \text { Punkte }\end{array}$ & $\begin{array}{l}\text { Diabetes mellitus } \\
4 \text { Punkte }\end{array}$ \\
\hline \multicolumn{4}{|l|}{ Auswertung } \\
\hline Typ 1 & < 10 Punkte: geringes Risiko & & \\
\hline Typ 2 & 10-20 Punkte: mittleres Risiko & & \\
\hline Tyр 3 & > 20 Punkte: hohes Risiko & & \\
\hline
\end{tabular}

\section{Klassifikationsmodelle}

\section{Morphologisch-deskriptive Klassifikation}

Die Klassifikation der Pseudarthrose ist ein Muss und Grundlage eines jeden Therapieplans. Im klassischen Sinne werden Pseudarthrosen entsprechend ihrer Morphologie in 4 Arten eingeteilt:

- hypertrophe Pseudarthrose

- atrophe Pseudarthrose

- Defektpseudarthrose

- Infektpseudarthrose

Hypertophe Pseudarthrosen zeichnen sich i.d.R. durch eine unzureichende mechanische Stabilität aus, was zu einer übermäßigen Kallusbildung im Frakturbereich führt. Allerdings bleibt die knöcherne Konsolidierung dabei aus. Atrophe Pseudarthrosen resultieren häufig aus einer schlechten lokalen biologischen Regenerationspotenz, meist durch eine verminderte Durchblutung des Frakturspalts und des umliegenden Knochens. Eine Atrophie der Frakturenden und damit fehlende Knochenbruchheilung ist die Konsequenz. Defektpseudarthrosen entstehen meist bei Hochrasanztraumata und komplexen offenen Frakturen (Mehretagenfrakturen und mehrfragmentäre Frakturen etc.), bei denen es entweder durch den Unfall oder durch die Erstoperation zum Verlust/Entnahme des Defektknochens kommt. Der oben beschriebene Frakturmechanismus führt daher auch häufig zur 4. Pseudarthrosenform: der Infektpseudarthrose. Diese entsteht vorwiegend bei offenen Frakturen mit Verlust der Weichteildeckung und dadurch eintretende Keime [14].

Neben diesen eher deskriptiven Grundlagen der Pseudarthroseneinteilung existiert eine Vielzahl an Klassifikationssystemen zur Analyse der Ätiologie und korrekten Therapieplanung. Leider gibt es bis heute auch hier keinen Goldstandard oder ein allumfassendes System. Allen Klassifikationssystemen zugrunde liegt eine mehr oder weniger detaillierte präoperative Analyse der Problematik. Dies ist essenziell, da nur so die hohe Misserfolgsrate der Pseudarthrosentherapie von bis zu $20 \%$ weiter reduziert werden kann und die entsprechende Behandlungsstrategie unmittelbar von der Ätiologie der Pseudarthrose abhängt [15]. Somit sollte jedes Klassifikationssystem 


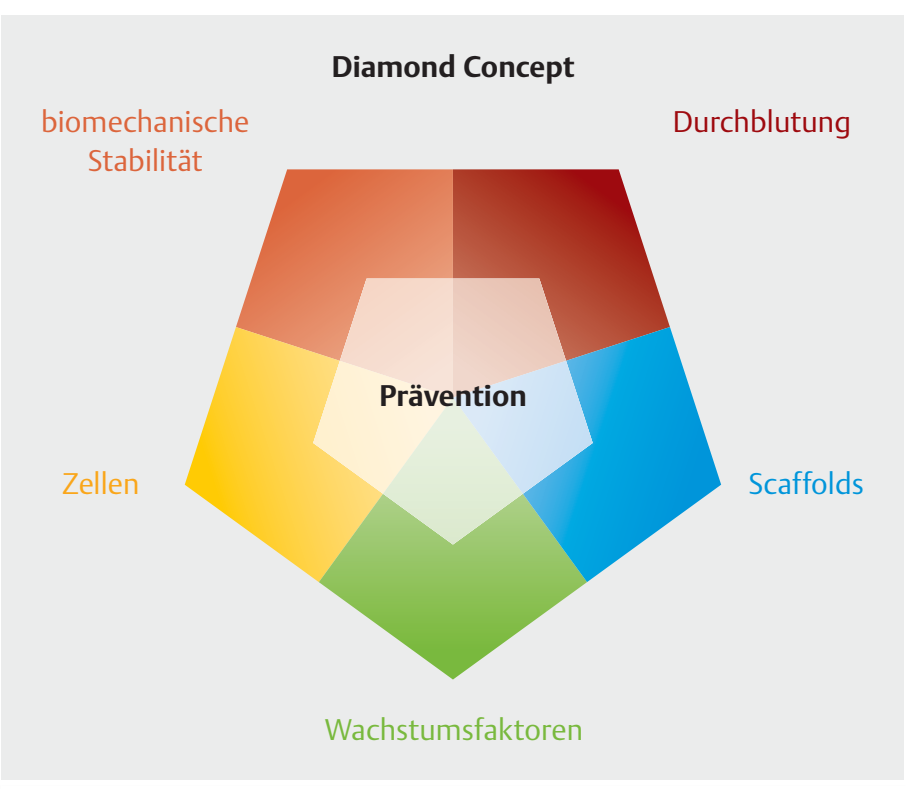

- Abb. 1 Diamantkonzept der Pseudarthrosenanalyse. Quelle: Schmidmaier G, Moghaddam A. Pseudarthrosen langer Röhrenknochen. Z Orthop Unfall 2015; 153: 659-676. dem Verständnis der Ursache einer Pseudarthrose auf den Grund gehen, um so das entsprechende Therapieverfahren der Kausalität entsprechend zu wählen. Als grundlegende Kernproblematiken einer Pseudarthrosenentstehung werden, mit Blick auf die Pathophysiologie der Knochenheilung, i.d.R. mechanische Instabilität, Distanz der Frakturfragmente, offene Frakturen und/oder ausgedehnter Weichteilschaden, Infektion oder Durchblutungsstörung angesehen [16]. Hilfreich in der ersten Beurteilung der Pseudarthrose vor Anwendung einer Klassifikation ist daher die Verwendung des Diamantkonzepts, um sich über die Rahmenbedingungen einen Überblick zu verschaffen ( $\triangleright$ Abb. 1) [13].

\section{Klassifikation nach Weber und Czech}

Die etablierte Klassifikation nach Weber und Czech von 1973 stützt sich auf radiologische Befunde und unterteilt Pseudarthrosen anhand ihrer Vitalität und des Heilungspotenzials [17]. Sie werden in biologisch reaktionsfähig ( $A$, vital und hypertroph) und biologisch inaktiv (B, avital und atroph) unterschieden. Die vitalen Pseudarthrosen werden dementsprechend weiter in hypertrophe (Elefantenfußpseudarthrose), mäßig hypertrophe (Pferdefußpseudarthrose) und oligotrophe Pseudarthrose unterteilt. Ihnen liegt i.d.R. eine mechanische Ursache aufgrund einer Instabilität der Osteosynthese zugrunde. Avitale Pseudarthrosen hingegen zeichnen sich meistens durch eine kompromittierte lokale Biologie (Durchblutungssituation, fehlende osteogene Potenz) aus. Hier werden 4 Subtypen unterschieden: Drehkeilpseudarthrose, Trümmerpseudarthrose, Defektpseudarthrose und atrophe/reaktionslose Pseudarthrose ( $\boldsymbol{A}$ Abb. 2) $[5,10]$.
Während hypertrophe Pseudarthrosen nach ausreichender Stabilisierung noch in der Lage sind eigenständig ausheilen zu können, müssen bei avitalen Pseudarthrosen zunächst wieder vitale lokale Verhältnisse geschaffen werden. Eine Ausheilung ist allein durch einen Verfahrenswechsel der Osteosynthese nicht möglich, sondern es bedarf des Einsatzes biologischer Augmentation (z. B. autologe Spongiosaplastik) resp. einer Infektsanierung, um eine Ausheilung zu ermöglichen. Eine konkrete Handlungsanweisung schließt sich jedoch nicht an die Klassifikation an.

\section{Non-Union Scoring System (NUSS) nach Calori}

Ein aktuelleres Klassifikationssystem stellt das NUSS aus dem Jahr 2008 dar, was neben der Pseudarthrosenanalyse auch eine Behandlungsempfehlung ableitet. Es beinhaltet neben der klassischen radiologischen Beurteilung wichtige klinische Aspekte und Einflussfaktoren. Calori und Mitarbeiter berücksichtigen neben den Hauptkriterien „Knochen“ („,bone“), „Weichteile“ („soft tissue“) und „patientenabhängige Faktoren“ („patient“) weitere Kerneinflussfaktoren (operative Versorgungsqualität, Medikamente etc.) und bewerten diese mittels eines Punktesystems. Der erzielte Gesamtscore addiert sich auf maximal 50 Punkte und wird am Ende verdoppelt. Hieraus leiten sich 4 Gruppen ab, um basierend auf dem ermittelten Score eine Behandlungsempfehlung zu geben ( $\triangleright$ Tab. 5) [15]. Diese Therapieempfehlungen lassen sich entsprechend vereinfacht wie folgt unterscheiden [10]:

1. < 25 Punkte: Instabilitätsproblem mit i.d.R. Wechsel des Osteosyntheseverfahrens, lokale Augmentation nicht zwingend erforderlich

2. 26-50 Punkte: Kombination aus biologischem und mechanischem Problem, Osteosyntheseverfahrenswechsel und biologische Augmentation erforderlich

3. 51-75 Punkte: spezialisierte Therapie mit ggf. Umgebungsfaktorenverbesserung (Rekanalisation Gefäße, Infektsanierung etc.), Verbesserung mechanischer und biologischer Voraussetzungen, komplexe Therapie mit Pseudarthrosenresektion, Spongiosaplastik oder Segmenttransport

4. > 75 Punkte: geringe Heilungsaussichten mit komplexer, langwieriger Therapie, häufig Amputation, Arthrodese oder Prothesenimplantation sinnvoller

Aus Sicht der Autoren sollte die dezidierte NUSS-Klassifikation als der aktuelle Goldstandard in der Einteilung der Pseudarthrose und der daraus abgeleiteten Therapieempfehlung genutzt werden. Durch die genaue Analyse ist eine exakte Therapieplanung möglich und dadurch können Heilungsraten mit bis zu $82-87 \%$ in den ersten 3 Gruppen erzielt werden $[15,18]$. Das Scoring-System ermöglicht die klare Unterscheidung komplexer Pseudarthrosen von einfachen Pseudarthrosen und erleichtert dadurch die Formulierung einer Therapiestrategie insbesondere unter der Berücksichtigung patientenindividueller Besonderheiten. Allerdings fehlt der NUSS-Klassifika- 


\section{vitale Pseudarthrose}
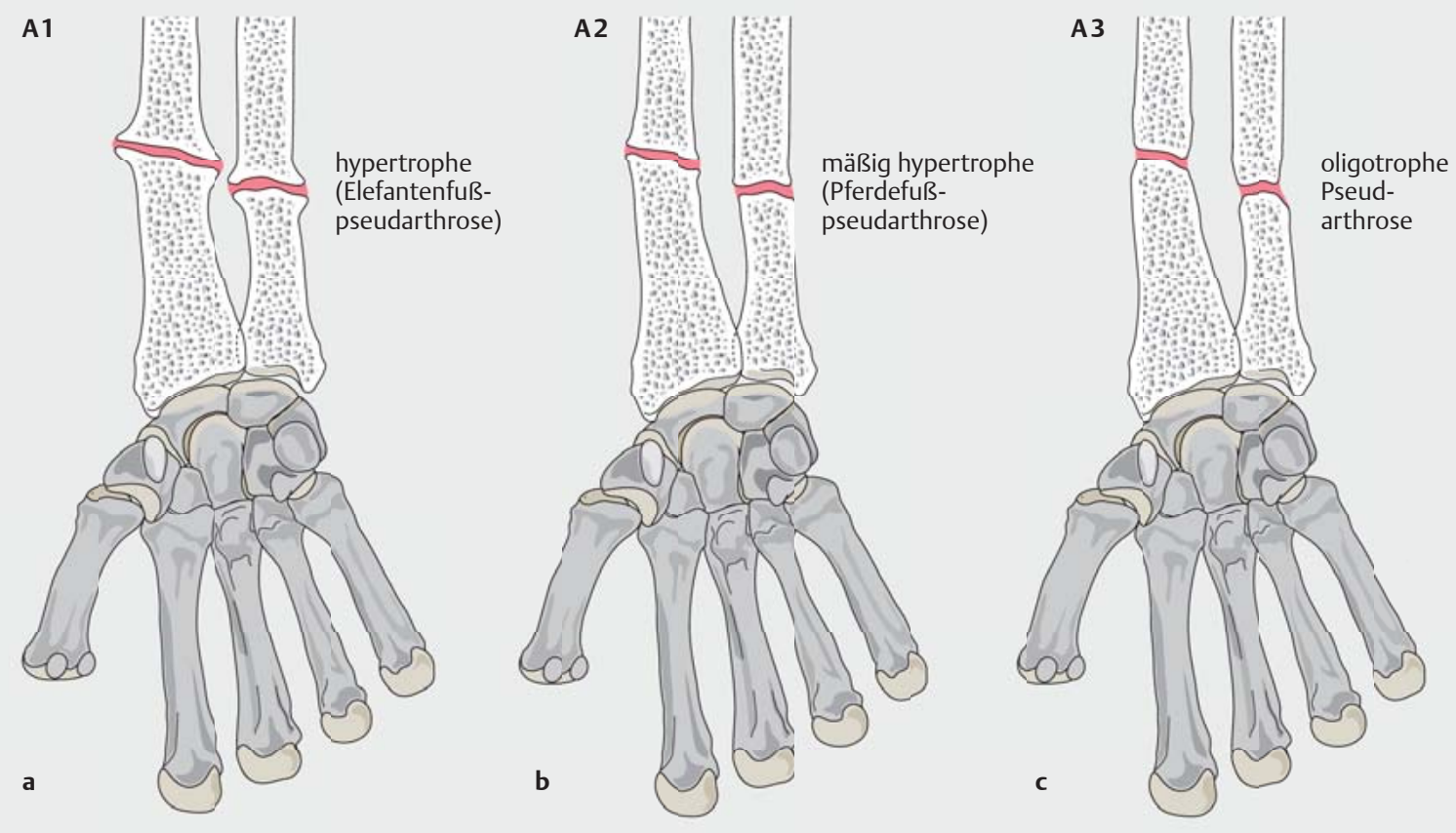

\section{avitale Pseudarthrose}

B1

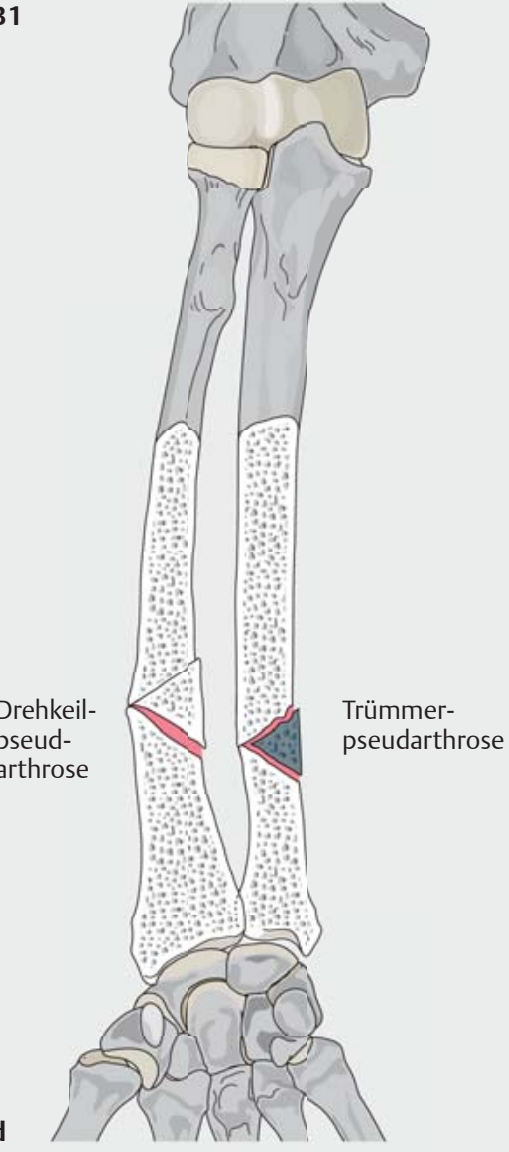

B2

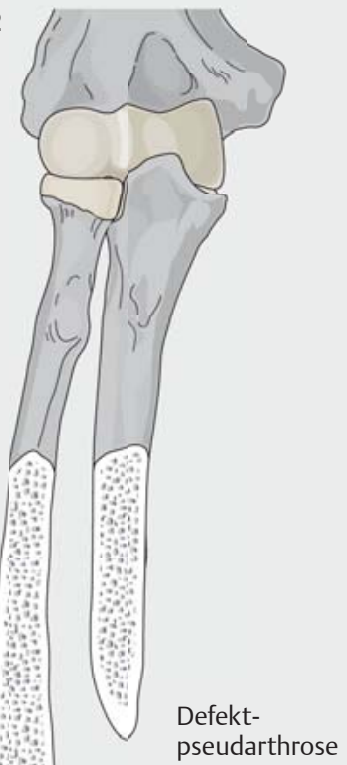

- Abb. 2 Klassifikation der Pseudarthrosen nach Weber und Czech. Hypertrophe Pseudarthrosen A1-A3. a Reaktiv, hypertroph.

b Kallusarm. c Kalluslos. Avitale Pseudarthrosen B1-B3. d Drehkeilpseudarthrosen. e Defektpseudarthrose. f Atroph. 
- Tab. 5 Non-Union Scoring System (NUSS) nach Calori mit Punktwerterrechnung [15].

\begin{tabular}{|c|c|c|c|}
\hline Knochen & Variable & Punkte & Maximalpunktzahl \\
\hline Knochenqualität & $\begin{array}{l}\text { gut } \\
\text { mäßig } \\
\text { schlecht } \\
\text { sehr schlecht }\end{array}$ & $\begin{array}{l}0 \\
1 \\
2 \\
3\end{array}$ & 3 \\
\hline $\begin{array}{l}\text { Traumamechanismus } \\
\text { (offen/geschlossen) }\end{array}$ & $\begin{array}{l}\text { geschlossen } \\
\text { erstgradig offen } \\
\text { zweitgradig offen } \\
\text { drittgradig offen }\end{array}$ & $\begin{array}{l}0 \\
1 \\
3 \\
5\end{array}$ & 5 \\
\hline $\begin{array}{l}\text { durchgeführte Operationen } \\
\text { am Knochen }\end{array}$ & $\begin{array}{l}\text { keine } \\
<2 \\
2-4 \\
>4\end{array}$ & $\begin{array}{l}1 \\
2 \\
3 \\
4\end{array}$ & 4 \\
\hline $\begin{array}{l}\text { Invasivität der durchgeführten } \\
\text { Operationen am Knochen }\end{array}$ & $\begin{array}{l}\text { minimalinvasiv } \\
\text { intramedulläres Verfahren } \\
\text { extramedulläres Verfahren } \\
\text { Knochentransplantation }\end{array}$ & $\begin{array}{l}0 \\
1 \\
2 \\
3\end{array}$ & 3 \\
\hline Stabilität der Primärosteosynthese & $\begin{array}{l}\text { stabil } \\
\text { instabil }\end{array}$ & $\begin{array}{l}0 \\
1\end{array}$ & 1 \\
\hline Klassifikation nach Weber und Czech & $\begin{array}{l}\text { hypertoph } \\
\text { oligotroph } \\
\text { atroph }\end{array}$ & $\begin{array}{l}1 \\
3 \\
5\end{array}$ & 5 \\
\hline Frakturreposition & $\begin{array}{l}\text { nicht anatomisch } \\
\text { anatomisch }\end{array}$ & $\begin{array}{l}0 \\
1\end{array}$ & 1 \\
\hline Knochendefekt & $\begin{array}{l}0,5-1 \mathrm{~cm} \\
1-3 \mathrm{~cm} \\
>3 \mathrm{~cm}\end{array}$ & $\begin{array}{l}2 \\
3 \\
5\end{array}$ & 5 \\
\hline \multicolumn{4}{|l|}{ Weichteilstatus } \\
\hline Weichteile & $\begin{array}{l}\text { intakt } \\
\text { Vernarbung } \\
\text { lokale Weichteildeckung } \\
\text { freie Lappenplastik } \\
\text { schlechte Durchblutung } \\
\text { Hautdefekte }\end{array}$ & $\begin{array}{l}0 \\
2 \\
3 \\
4 \\
5 \\
6\end{array}$ & 6 \\
\hline \multicolumn{4}{|l|}{ patientenbezogene Risikofaktoren } \\
\hline ASA-Score & $\begin{array}{l}1-2 \\
3-4\end{array}$ & $\begin{array}{l}0 \\
1\end{array}$ & 1 \\
\hline Diabetes mellitus & $\begin{array}{l}\text { nein } \\
\text { ja, gut eingestellt } \\
\text { ja, schlecht eingestellt }\end{array}$ & $\begin{array}{l}0 \\
1 \\
2\end{array}$ & 2 \\
\hline $\begin{array}{l}\text { Blutwerte (Leukozyten, } \\
\text { Blutsenkungsgeschwindigkeit, } \\
\text { C-reaktives Protein) }\end{array}$ & $\begin{array}{l}\text { Leukozyten }>12000 \\
\text { BSG }>20 \\
\text { CRP }>20\end{array}$ & $\begin{array}{l}1 \\
1 \\
1\end{array}$ & 3 \\
\hline lokaler Infektionsbefund & $\begin{array}{l}\text { sauber } \\
\text { infiziert } \\
\text { septisch }\end{array}$ & $\begin{array}{l}0 \\
1 \\
4\end{array}$ & 4 \\
\hline Medikamente & $\begin{array}{l}\text { Steroide } \\
\text { nicht steroidale Antirheumatika (NSAR) }\end{array}$ & $\begin{array}{l}1 \\
1\end{array}$ & 2 \\
\hline Rauchen & $\begin{array}{l}\text { nein } \\
\text { ja }\end{array}$ & $\begin{array}{l}0 \\
5\end{array}$ & 5 \\
\hline
\end{tabular}



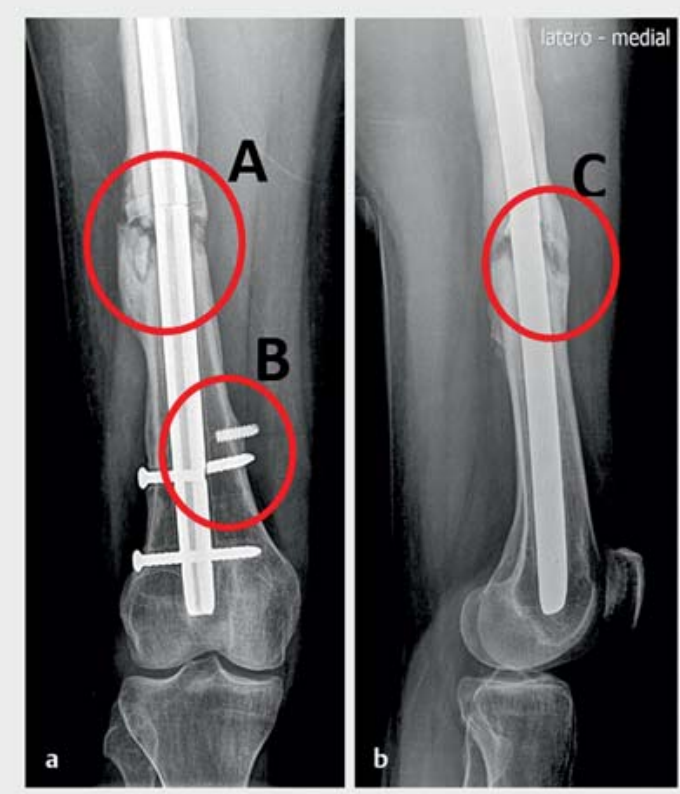

- Abb. 3 Beispiel einer Infektpseudarthrose mit Mischbild aus atropher und hypertropher Morphologie sowie Sequesternachweise $(\mathrm{a}, \mathrm{c})$, Materialbruch aufgrund von $\mathrm{Er}$ müdung der Verriegelungsbolzen sowie des Nagels $(\mathbf{a}, \mathbf{b})$.

tion eine Risiko-Nutzen-Abwägung, was in Anbetracht des Stellenwerts der Lebensqualität für den Patienten eine Limitation darstellt.

\section{Weitere Klassifikationssysteme}

Der Vollständigkeit halber wird die Klassifikation nach Paley ebenfalls erwähnt. Diese findet im Speziellen bei Tibiapseudarthrosen Anwendung und beinhaltet eine weitere Differenzierung entsprechend den Knochendefekten/-verlusten $<1 \mathrm{~cm}$ (Typ A) und > $1 \mathrm{~cm}$ (Typ B) [19]. Sie kann auch auf andere Knochen angewendet werden, ist jedoch aus Sicht der Autoren nicht dezidiert genug und ohne wesentliche Konsequenz. Eine weitere Klassifikationsmöglichkeit besteht nach Cierny-Mader. Die Pseudarthrose wird entsprechend ihrer aseptischen bzw. septischen Genese unterschieden und beurteilt den physiologischen Status des Patienten, um die notwendige Therapie einer Risiko-Nutzen-Analyse zu unterziehen [20]. Diese ist insbesondere hinsichtlich der Lebensqualitätsbeurteilung eine gute Evaluation und hilfreich für den Arzt und den Patienten, jedoch ebenfalls in ihrer Gesamtheit sehr limitiert.

Von allen gängigen Klassifikationssystemen abzugrenzen ist die Beurteilung der Infektpseudarthrose, die sowohl mit einer hyper- als auch atrophen Knochenreaktion ein- hergehen kann ( $\triangleright$ Abb. 3). Insbesondere bei jungen, immunkompetenten Patienten kann eine hypertrophe Infektpseudarthrose auf Basis einer Low-Grade-Infektion („Infektkallus“) als reguläre hypertrophe Pseudarthrose fehlgedeutet werden - eine große diagnostische Herausforderung, da in der Literatur zunehmend Evidenz für eine Pseudarthrosenentstehung basierend auf einer Infektgenese gezeigt wird. Sie ist sozusagen der „Wolf im Schafspelz" und kann zu einer falschen Therapieplanung verleiten [3, 5, 9]. Eine weitere Limitation aller erwähnten Klassifikationssysteme ist die fehlende strukturierte Beurteilung möglicher begleitendender Deformitäten, wie der Achs-, Rotations- und Längenverhältnisse. Hier sollten die gleichen Konzepte wie bei fehlverheilten Frakturen angewandt werden, da nur bei nahezu anatomischer Rekonstruktion ein problemloses Ausheilen zu erwarten ist.

\section{Fazit}

Die Definition einer verzögerten Knochenheilung resp. Pseudarthrose ist als dynamische Beurteilung des Heilungsverlaufs zu verstehen und muss neben radiologischen vor allem auch patientenindividuelle Faktoren berücksichtigen. Der Zeitraum nach Trauma stellt hierbei nach wie vor eine gute Orientierung für den behandelnden Arzt dar, darf jedoch nicht als pauschales Diagnostikum Anwendung finden. Die Kombination aus radiologischem Verlauf, klinischer Symptomatik und patientenindividuellen Faktoren ist der Schlüssel in der Therapieplanung. Hierbei ermöglichen die verschiedenen Klassifikationssysteme eine mehr oder weniger strukturierte und detaillierte Analyse der zugrunde liegenden Pathologie der Knochenheilungsstörung. Das NUSS-Klassifikationssystem kann darüber hinaus eine mögliche Therapieempfehlung abgeben und somit den behandelnden Arzt unterstützen. Die korrekte Beurteilung von Pseudarthrosen kann dadurch erleichtert werden, allerdings sind die Übergänge der einzelnen Formen fließend. Die zunehmende Rate an Low-Grade-Infektionen und sog. hypertrophen Infektpseudarthrosen stellt eine diagnostische Herausforderung dar. Es ist daher unumgänglich, die Therapie von Knochenheilungsstörungen in spezialisierte Hände zu geben, um ein individuelles Therapiekonzept zu erstellen und die operative Strategie auf den Patienten und auch seinen langen Genesungsweg abzustimmen. Hierbei können Scoring-Systeme zur Risikoprofileinschätzung hilfreich sein und in Zukunft zusätzlich in die Klassifikationssysteme mit eingearbeitet werden. Einen konkreten Zeitpunkt für eine pauschale Revisionsnotwendigkeit zu definieren, ist jedoch basierend allein auf den Klassifikationssystemen nicht zielführend. 


\section{Autorinnen/Autoren}

\section{Karl Friedrich Braun}

PD Dr. med., Facharzt für Unfallchirurgie und Orthopädie, Spezielle Unfallchirurgie, Oberarzt der Klinik und Poliklinik für Unfallchirurgie, Klinikum rechts der Isar, Technische Universität München, Fakultät für Medizin

\section{Marc Hanschen}

PD Dr. med., Facharzt für Unfallchirurgie und Orthopädie, Spezielle Unfallchirurgie, Oberarzt der Klinik und Poliklinik für Unfallchirurgie, Klinikum rechts der Isar, Technische Universität München, Fakultät für Medizin

\section{Peter Biberthaler}

Prof. Dr. med., Direktor der Klinik und Poliklinik für Unfallchirurgie, Klinikum rechts der Isar, Technische Universität München, Fakultät für Medizin

\section{Korrespondenzadresse}

\section{PD Dr. med. Karl F. Braun}

Klinikum rechts der Isar

Technische Universität München

Ismaninger Straße 22

81675 München

Tel.: 089/4140-9383

Fax: 040/4140-4890

karl.braun@charite.de

\section{Literatur}

[1] Einhorn TA, Lee CA. Bone regeneration: new findings and potential clinical applications. J Am Acad Orthop Surg 2001; 9: 157-165

[2] Ai-Aql ZS, Alagl AS, Graves DT et al. Molecular mechanisms controlling bone formation during fracture healing and distraction osteogenesis. J Dental Res 2008; 87: 107-118

[3] Biberthaler P, van Griensven M. Knochendefekte und Pseudarthrosen. Berlin: Springer; 2017. doi:10.1007/978-3-64244991-8

[4] Mills LA, Simpson AH. The relative incidence of fracture nonunion in the Scottish population (5.17 million): a 5-year epidemiological study. BMJ Open 2013. doi:10.1136/bmjopen2012-002276

[5] Everding J, Roßlenbroich S, Raschke MJ. Pseudarthrosen der langen Röhrenknochen. Chirurg 2018; 89: 73-88. doi:10.1007/s00104-017-0547-4
[6] Zimmermann G, Müller U, Löffler C et al. Therapieerfolg bei atrophen Tibiaschaftpseudarthrosen. Unfallchirurg 2007; 110: 931-938

[7] Moghaddam-Alvandi A, Zimmermann G, Hammer K et al. Cigarette smoking influences the clinical and occupational outcome of patients with tibial shaft fractures. Injury 2013; 44: 1670-1671

[8] Forrest CR, Pang CY, Lindsay WK. Pathogenesis of ischemic necrosis in random-pattern skin flaps induced by long-term low-dose nicotine treatment in the rat. Plast Reconstr Surg 1991; 87: 518-528

[9] Steinhausen E, Glombitza M, Böhm HJ et al. Pseudarthrosen: Von der Diagnose bis zur Ausheilung. Unfallchirurg 2013; 116: 633-649

[10] Breer S, Auerwald M, Fuchs S et al. Versorgung von Pseudarthrosen der langen Röhrenknochen. Trauma Berufskrankh 2015; 17: $233-243$

[11] Tzioupis C, Giannoudis PV. Prevalence of long-bone nonunions. Injury 2007; 38 (Suppl. 2): S3-S9

[12] Barnes GL, Kostenuik PJ, Gerstenfeld LC et al. Growth factor regulation of fracture repair. J Bone Mineral Res 1999; 14: 1805-1815

[13] Schmidmaier G, Moghaddam A. Pseudarthrosen langer Röhrenknochen. Z Orthop Unfall 2015; 153: 659-676

[14] Calori GM, Albisetti W, Agus A et al. Risk factors contributing to fracture non-unions. Injury 2007; 38 (Suppl. 2): S11-S18

[15] Calori GM, Phillips M, Jeetle $S$ et al. Classification of non-union: need for a new scoring system? Injury 2008; 39 (Suppl.2): S59-S63

[16] Bhandari M, Guyatt GH, Swiontkowski MF et al. A lack of consensus in assessment of fracture healing among orthopedic surgeons. J Orthop Trauma 2005; 16: 562-566

[17] Weber BG, Czech O. Pseudarthrosen - Pathophysiologie, Biomechanik, Therapie, Ergebnisse. Bern, Stuttgart, Wien: Huber; 1973

[18] Calori GM, Colombo M, Mazza EL. Validation of the Non-Union Scoring System in 300 long bone non-unions. Injury 2014; 45 (Suppl. 6): S93-S97

[19] Paley D, Catagni MA, Argnani F et al. llizarov treatment of tibial nonunions with bone loss. Clin Orthop Relat Res 1989; (241): 146-165

[20] Cierny G 3rd, Mader JT, Penninck JJ. A clinical staging system for adult osteomyelitis. Clin Orthop Relat Res 2003; (414): 7 24

Bibliografie

DOI https://doi.org/10.1055/a-0889-1013 OP-JOURNAL 2019; 35: 217-224 @ Georg Thieme Verlag KG Stuttgart · New York ISSN 0178-1715 